\title{
Distribution of type III secretion gene clusters in Burkholderia pseudomallei, B. thailandensis and B. mallei
}

\author{
LUCILLE RAINBOW, C. ANTHONY HART and CRAIG WINSTANLEY
}

Department of Medical Microbiology and Genitourinary Medicine, University of Liverpool, Duncan Building, Daulby Street, Liverpool L69 3GA, UK

\begin{abstract}
Burkholderia pseudomallei, the causative agent of melioidosis, carries a cluster of genes closely related in organisation to the type III secretion (TTS) system gene clusters of the plant pathogens Ralstonia solanacearum and Xanthomonas spp. The TTS gene cluster (TTS1) is present only in B. pseudomallei and not in avirulent $B$. thailandensis. Adjacent to the gene cluster encoding putative secreton structural proteins lie a number of open reading frames (ORFs) encoding putative proteins with little or no homology to known proteins, with the exception of one predicted protein with homology to Pseudomonas syringae HrpK. In both $R$. solanacearum and Xanthomonas spp., genes in this location encode secreted effector proteins. RT-PCR analysis indicated that TTS genes, including two of these ORFs, are expressed in broth at $37^{\circ} \mathrm{C}$. Analysis of genome sequence data identified a second cluster of TTS genes (TTS2) present in both $B$. pseudomallei and $B$. mallei (99\% identity). However, $B$. mallei appears to lack the TTS1 gene cluster. PCR assays indicated that TTS2 was also present in $B$. thailandensis. TTS1 and TTS2 are similar in gene organisation, but nucleotide sequences are sufficiently divergent to suggest that the two TTS systems may have different roles.
\end{abstract}

\section{Introduction}

Burkholderia pseudomallei is the causative agent of melioidosis, a disease that can manifest itself as an acute, subacute or chronic infection $[1,2]$. The acute form of the disease accounts for a significant proportion of often fatal, community-acquired septicaemia in areas of south-east Asia and Australia. B. pseudomallei also causes a subacute febrile illness leading to systemic abscess formation involving various organs. The chronic form of the disease is evident from discovery at autopsy or by subsequent activation causing other forms of the disease. It is known that B. pseudomallei can enter a dormant state following initial infection or recovery from clinical disease [3], and that dormant cells can be triggered, leading to relapse or the onset of acute melioidosis [4]. The mechanisms underlying these different clinical manifestations and the triggers causing the more serious forms of the disease are poorly understood.

Received 5 Sept. 2001; revised version accepted 7 Dec. 2001.

Corresponding author: Dr C. Winstanley (e-mail: C. Winstanley@liverpool.ac.uk).
It has been suggested that environmental $B$. pseudomallei isolates can be separated on the basis of a number of factors into two biotypes, best defined by the ability to assimilate arabinose. There is evidence that B. thailandensis ( $\mathrm{Ara}^{+}$strains) [5] are avirulent whereas $\mathrm{Ara}^{-}$strains constitute melioidosis-causing 'true' B. pseudomallei [6]. It is possible to discriminate between the two biotypes by molecular approaches such as $\mathrm{fliC}$ variation [7,8] or multiplex PCR assays [9]. Furthermore, differences in genomic macrorestriction patterns between the two biotypes have been reported [10]. As the mechanisms of B. pseudomallei virulence are poorly understood, there is considerable interest in the identification of the specific factors determining the greater virulence of $B$. pseudomallei. Indeed, subtractive hybridisation has been used to identify some virulence genes present in B. pseudomallei but absent from B. thailandensis [11].

Differences in virulence between closely related bacterial pathogens are often attributable to the presence of pathogenicity islands (PIs) in virulent strains. Type III secretion (TTS) systems have been implicated in the pathogenicity of several gram-negative bacterial pathogens, including intracellular bacteria such as Shigella 
spp. $[12,13]$. The genes encoding TTS systems and secreted effector proteins are associated with PIs and, therefore, are prime candidates for involvement in the greater virulence of some strains over other related strains.

A previous study identified, in B. pseudomallei, a cluster of putative genes homologous to those encoding HpaP, HrcQ (HrpQ), HrcR (HrpT), HrcS (HrpU) and HrpV, all TTS-associated proteins in the plant pathogen Ralstonia solanacearum [14]. A further study, based on the detection of a single locus, reported a link between the presence of putative TTS system genes and the $\mathrm{Ara}^{-}$phenotype, the more virulent biotype of $B$. pseudomallei [15]. There was one exception to the link, a B. thailandensis strain (E27) which was positive by both PCR and dot-blot assay.

This study reports the nucleotide sequence of the entire putative structural region of the B. pseudomallei TTS1 gene cluster, identifies ORFs that may encode potential effectors and provides evidence that TTS genes are expressed. The study also presents more comprehensive evidence of the link between TTS1 genes and the $\mathrm{Ara}^{-}$ biotype and reports evidence for a second TTS gene cluster (TTS2) common to B. pseudomallei and $B$. mallei, the causative agent of glanders.

\section{Materials and methods}

\section{Bacterial strains}

The strains used in this study have been described previously [15] and are listed in Table 1. B. pseudomallei and $B$. thailandensis strains were maintained on nutrient agar.

\section{Nucleotide sequence of TTS gene PI}

Cosmid clones c503-23, c503-5 and c503-V1 were previously identified as containing a TTS gene cluster [14], for which we propose the name TTS1. Fragments generated by HindIII digestion were subcloned from these cosmid clones into HindIII-digested pUC19 (Helena Biosciences). These and other subclones were sequenced by primer-walking with vector and internal oligonucleotide primer sequences.

\section{Computer analyses}

Nucleotide sequence alignments, $\mathrm{G}+\mathrm{C}$ mol\% values, determination of amino acid composition, predicted protein mass and alignments of predicted proteins with other related proteins (retrieved from EMBL, GenBank, PIR or SwissProt [16]) were performed with BESTFIT, GAP, PILEUP, PRETTY, COMPOSITION, PEPTIDESORT and FASTA from the GCG sequence analysis software package (Genetics Computer Group, University of Wisconsin). Hydrophobicity profiles and predictions of membrane-spanning regions were obtained with the PEPTIDESTRUCTURE and PEPPLOT programmes, employing the hydropathy measure of Kyte and Doolittle [17] and the measure of helical hydrophobic moment [18]. The programme MOTIFS was used to identify motifs within the predicted protein sequences. BLAST searches were conducted via the site http://www.ncbi.nlm.nih.gov/blast/blast.cgi [19]. Genome sequence data for $B$. pseudomallei, used to complete orf13, and for TTS2, were obtained from the Sanger Centre website http://www.sanger.ac.uk/ Projects/B_pseudomallei/. These sequence data were produced by the B. pseudomallei Sequencing Group at the Sanger Centre and can be obtained from $\mathrm{ftp}: / /$ ftp.sanger.ac.uk/pub/pathogens/bps/. Preliminary sequence data for the genome sequence of $B$. mallei were obtained from the Institute for Genomic Research website at http://www.tigr.org. Searches of these data were conducted via the BLASTX or TBLASTN facilities provided at these websites.

\section{Dot-blot and PCR assays}

Genomic DNA extraction from B. pseudomallei and dot-blot hybridisation of genomic DNA (c. $0.25 \mu \mathrm{g}$ ) were performed as described previously [15]. Digoxigenin-11-2'-dUTP (DIG) (Roche)-labelled probes were made either by PCR amplification with c503-5 or c50323 DNA as template in the presence of $60 \mu \mathrm{M}$ DIG, as described previously [15], or by labelling amplicons

Table 1. Strains used in this study and results of TTS1 gene PCR/dot-blot assays

\begin{tabular}{|c|c|c|c|c|c|c|c|}
\hline \multirow[b]{2}{*}{ Strains } & \multirow{2}{*}{$\begin{array}{l}\text { orfl1 } \\
\text { PCR }\end{array}$} & \multicolumn{2}{|c|}{ orf7 } & \multicolumn{2}{|c|}{ bpscJ } & \multirow{2}{*}{$\frac{\text { orf4/orf5 }}{\text { Dot-blot }}$} & \multirow{2}{*}{$\frac{\text { orf1-bpscQ gap }}{\text { Dot-blot }}$} \\
\hline & & PCR & Dot-blot & PCR & Dot-blot & & \\
\hline B. pseudomallei & & & & & & & \\
\hline $\begin{array}{l}\text { E503, E504, E505, E506, } 204 \text { (E955), } \\
576 \text { (E957), E25 (E958), E8 (E960) }\end{array}$ & + & + & + & + & + & + & + \\
\hline B. thailandensis & & & & & & & \\
\hline E27 (E956) & - & - & - & $+/-$ & - & - & - \\
\hline $\begin{array}{l}\text { E82 (E959), E32, E111, E125, E132, } \\
\text { E135, E202, E216, E251, E253, } \\
\text { E254, E255, E260 }\end{array}$ & - & - & - & - & - & - & - \\
\hline Neisseria meningitidis $\mathrm{C} 311$ & ND & ND & - & ND & - & - & - \\
\hline
\end{tabular}

ND, not done. 
directly with the DIG-High Prime labelling system (Roche) and following the manufacturer's instructions.

Oligonucleotide primers (Genosys) used in PCR or RTPCR assays and for the labelling of probes are listed in Table 2 along with the annealing temperatures used. Typically, genomic DNA (1 $\mu \mathrm{l})$ was used directly in $25-\mu 1$ volumes containing 2 units of Dynazyme (Flowgen), $200 \mathrm{nM}$ of each primer, $1 \times$ Dynazyme buffer and $100 \mu \mathrm{M}$ nucleotides (dATP, dCTP, dGTP, dTTP). Amplifications were performed in an Eppendorf MasterCycler thermal cycler for 30 cycles consisting of $95^{\circ} \mathrm{C}(1 \mathrm{~min})$, annealing temperature $(1 \mathrm{~min})$ and $72^{\circ} \mathrm{C}$ $(2 \mathrm{~min})$ with an additional extension time at $72^{\circ} \mathrm{C}$ ( $10 \mathrm{~min}$ ) following completion of the 30 cycles. At the end of the amplification, 5- $\mu 1$ samples were subjected to electrophoresis on a standard agarose $1.0 \% \mathrm{w} / \mathrm{v}$ gel to confirm the presence of an amplified product.

\section{RNA extraction and RT-PCR}

B. pseudomallei E503 (also known as strain 10-705) and B. thailandensis E27 were cultured in Luria broth at $37^{\circ} \mathrm{C}$ with and without the addition of $10 \mathrm{~mm}$ nitrilotriacetic acid (NTA) until they reached exponential growth $\left(\mathrm{A}_{600}\right.$ of $c$. 0.5). Cells were harvested by centrifugation and RNA was extracted with an RNeasy kit (Qiagen). Total RNA was treated with DNAase following the manufacturer's instructions (Life Technologies) and reverse transcribed into cDNA with $c$. $1 \mu \mathrm{g}$ of total RNA and $200 \mathrm{ng}$ of random hexamers (Life Technologies) in the presence of RNaseOUT ribonuclease inhibitor $(2 \mathrm{U} / \mu \mathrm{l}$; Life Technologies). PCR amplification was performed as described earlier. The flagellin gene $(f i C)$ was amplified with the primers BC6E and BCR14 as described previously [7].

RT-PCR products visualised after agarose $1 \% \mathrm{w} / \mathrm{v}$ gel electrophoresis were often faint. To ensure that there was convincing discrimination between positive and negative PCR assays, gels were Southern blotted by standard procedures and probed with equivalent labelled PCR products generated from genomic DNA. After purification through Microspin S-400 HR columns (Amersham Pharmacia Biotech), PCR products were labelled with DIG with a DIG-High Prime labelling system (Roche).

\section{Accession number}

The B. pseudomallei TTS1 system gene cluster nucleotide sequence reported in this paper can be found under the GenBank accession no. AF074878.

\section{Results}

\section{Map of the TTS genes}

A map of the strain E503 region sequenced from three overlapping cosmid clones is presented in Fig. 1. It has been possible to extend further to complete orfl 3 with additional sequence data produced by the B. pseudomallei Sequencing Group at the Sanger Centre (obtained from ftp://ftp.sanger.ac.uk/pub/pathogens/ bps). The region spanning bpscC to orf 3 bears a close resemblance in gene organisation to the TTS gene clusters encoding the Hrp secretons of the plant pathogens $R$. solanacearum $[20,21]$ and Xanthomonas spp. (X. campestris pv. vesicatoria or $X$. oryzae pv. oryzae) [22-24].

\section{Protein sequence alignments and motifs}

Table 3 indicates the sequence similarities of the equivalent predicted proteins from B. pseudomallei and $R$. solanacearum. In most cases there was good alignment between the predicted proteins of the two organisms. BLASTP searches indicated that most of

Table 2. Oligonucleotide primers used in this study

\begin{tabular}{|c|c|c|}
\hline $\begin{array}{l}\text { Target DNA } \\
\text { (amplicon size) }\end{array}$ & Oligonucleotide primer sets for PCR & $\begin{array}{c}\text { Annealing } \\
\text { temperature } \\
\left({ }^{\circ} \mathrm{C}\right)\end{array}$ \\
\hline orf11 & PM122: 5'-ATCGCCAAATGCCGGGTTTC & 55 \\
\hline (706 bp) & PM123: 5'-GTGCATCCATTCATCAAAG & \\
\hline orf10 & PM121: 5'-AGGCCCGTTACCCGATTG-3' & 60 \\
\hline$(219 \mathrm{bp})$ & PM127: 5'-CGACAGGACTGGAGAAAG-3' & \\
\hline orf7 & PM73: 5'-GACGTACGAACCGAACAA-3' & 60 \\
\hline (1059 bp) & PM80: 5'-AAGAAGTACGCGGCATTC-3' & \\
\hline bpscJ & PM98: 5'-TGACGGTTTGGCTGTTTG-3' & 55 \\
\hline (481 bp) & PM99: 5'-GATGGACTGCAAAGAATG-3' & \\
\hline orf4/orf5 & PM84: 5'-ATCCTGTCCGAGGTTTGC-3' & 50 \\
\hline$(646 \mathrm{bp})$ & PM95: 5'-ACATTCACTTCACTCAGG-3' & \\
\hline orf1-bpscQgap* & PMXBA3: 5'-TGCATCTAGAATTCGGTTCGACGTTCCC-3' & 65 \\
\hline$(490 \mathrm{bp})$ & PMBAM: 5'-ATGTGGATCCGATCGACAGTTCAATCACCG-3' & \\
\hline orf13 & SEPCF: $5^{\prime}$-TGAGAGCAAACCGTAATA-3' & 48 \\
\hline$(722 \mathrm{bp})$ & SEPCR: 5'-AAGGCTCATCCAGAACGTTC-3' & \\
\hline bpscU2 (TTS2) & TTS2UF: 5'-TGGCCGAAGAGAAAACCGAA-3' & 63 \\
\hline (488 bp) & TTS2UR2: 5'-AAACAGCGAGGTCATCATC-3' & \\
\hline SopE homologue & TTS3F: 5'-ATGACTTACAACCCGAGA-3' & 55 \\
\hline (TTS3) (608 bp) & TTS3R: 5'-CTGAAGCTCGCGATACTC-3' & \\
\hline
\end{tabular}

*PMXBA3 and PMBAM contain terminal restriction sites that are not relevant to this study. 


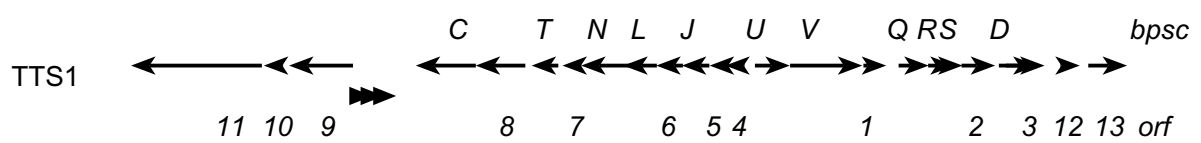

TTS2
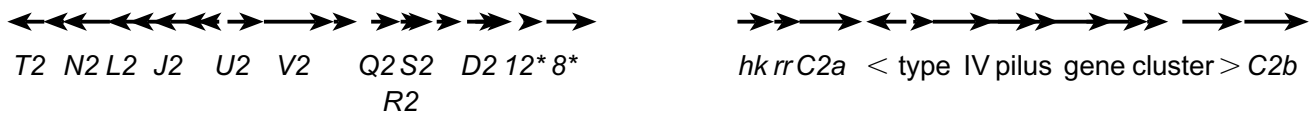

Fig. 1. Map of the TTS1 and TTS2 gene cluster. The location and lengths of predicted genes are shown. Arrows indicate the direction of transcription. The location of an imperfectly repeated sequence in TTS1 is also indicated. TTS2 genes have been labelled according to comparison with equivalent TTS1 genes. $12^{*}$ and $8^{*}$ indicate ORFs with homology to TTS1 orf12 and orf8 respectively. Genes with homology to histidine kinases $(h k)$ and response regulators $(r r)$ from known two-component regulatory systems are also indicated. Only genes with predicted protein sequences showing significant homology to proteins in the database are shown. The gap between orf $8^{*}$ and $h k$ is to scale but may contain other ORFs. The organisation of TTS2 genes is based on data obtained from the unfinished B. pseudomallei and B. mallei genome sequence projects.

Table 3. Properties of predicted TTS1 proteins and comparison with equivalent $R$. solanacearum and TTS2 proteins

\begin{tabular}{lccll}
\hline Protein* & $\begin{array}{c}\text { R. solanacearum } \\
\text { \% similarity/\% identity }\end{array}$ & $\begin{array}{c}\text { TTS2 } \\
\text { \% similarity/\% identity }\end{array}$ & $\begin{array}{l}\text { Yersinia } \\
\text { homologue }\end{array}$ & Predicted location/properties \\
\hline BpscC (HrpA/HrcC) & $56.2 / 45.8$ & $66.4 / 54.1(\mathrm{C} 2 \mathrm{~b})$ & YscC & Outer-membrane secretin \\
& & $39.2 / 29.6(\mathrm{C} 2 \mathrm{a})$ & & None \\
Orf8 (HrpB) & $47.7 / 36.1$ & $50.7 / 42.1$ & Transcriptional activator \\
BpscT (HrpC/HrcT) & $50.6 / 41.4$ & $56.5 / 48.5$ & YscT & Inner membrane \\
Orf7 (HrpD) & $41.4 / 29.9$ & $40.7 / 33.5$ & None & Unknown \\
BpscN (HrpE/HrcN) & $71.5 / 63.5$ & $79.9 / 72.2$ & YscN & ATPase \\
BpscL (HrpF) & $41.0 / 32.1$ & $57.3 / 46.4$ & YscL & Unknown \\
Orf6 (HrpH) & $37.0 / 33.0$ & $40.9 / 35.6$ & None & Unknown \\
BpscJ (HrpI/HrcJ) & $61.5 / 53.2$ & $64.5 / 59.7$ & Ysc & Lipoprotein \\
Orf5 (HrpJ) & $35.5 / 25.8$ & $40.2 / 31.5$ & None & Unknown \\
Orf4 (HrpK) & $41.0 / 30.8$ & $42.9 / 35.4$ & None & Unknown \\
BpscU (HrpN/HrcU) & $59.8 / 49.3$ & $59.5 / 47.3$ & YscU & Inner membrane \\
BpscV (HrpO/HrcV) & $69.3 / 61.1$ & $77.6 / 71.2$ & YscV (LcrD) & Inner membrane \\
Orf1 (HpaP) & $37.6 / 29.4$ & $43.7 / 39.2$ & None & Unknown \\
BpscQ (HrpQ/HrcQ) & $40.4 / 29.8$ & $47.5 / 42.0$ & YscQ & Translocase \\
BpscR (HrpT/HrcR) & $74.4 / 66.0$ & $82.0 / 73.7$ & YscR & Inner membrane \\
BpscS (HrpU/HrcS) & $64.0 / 55.8$ & $70.1 / 57.5$ & YscS & Inner membrane \\
Orf2 (HrpV) & $33.8 / 26.8$ & $35.3 / 29.9$ & None & Unknown \\
BpscD (HrpW) & $40.9 / 32.7$ & $49.8 / 43.8$ & YscD & Inner membrane \\
Orf3 (HrpX) & $36.4 / 29.9$ & $47.6 / 37.8$ & None & Unknown \\
\hline
\end{tabular}

* Protein designations shown in parentheses relate to $R$. solanacearum Hrp proteins. Both the original and currently accepted designations for these proteins are given.

$\dagger \%$ similarity $/ \%$ identity values were calculated by GAP/BESTFIT with the following parameters: gap creation penalty 8 , gap extension penalty 2.

Orf6 and HrpH do not align well. The figures presented were calculated by BESTFIT over a sequence of 113 amino acids.

${ }^{\S}$ BpscJ data were calculated for the whole predicted peptide sequence not for the protein predicted after cleavage of the lipoprotein signal sequence.

the B. pseudomallei predicted TTS1 proteins matched best with homologues from either $R$. solanacearum or Xanthomonas spp. The exceptions to this were Orf2, Orf3, Orf6 and Orf7, for which the BLAST searches identified no significant similarity. Similarity/identity values were particularly high for the 11 proteins belonging to families whose members are known to be conserved between TTS systems (BpscC, BpscD, BpscN, BpscL, BpscJ, BpscQ, BpscR, BpscS, BpscT, $\mathrm{Bpsc}$ and $\mathrm{BpscV}$ ). There was also strong homology between Orf8 and $R$. solanacearum HrpB, a transcriptional activator responsible for regulating TTS genes in $R$. solanacearum. Further analysis with the NCBI
Conserved Domain Database at http://www.ncbi.nlm. nih.gov/Structure/cdd/cdd.shtml indicated a highly significant match with bacterial regulatory helix-turn-helix proteins of the AraC family in the C-terminal domain (Orf8 394-483). Members of the AraC-XylS family are basic. Whereas HrpB has a pI value of 8.56, Orf8 is only weakly basic, with a pI of 7.06. Even amongst those proteins exhibiting lower similarity/identity values there are similarities in predicted $\mathrm{pI}$ and mol. wt values (data not shown). The least convincing match between the two TTS systems was found with Orf6, which does not align well with $\mathrm{HrpH}$, although the predicted $\mathrm{pI}$ values are similar. 
The predicted properties of $\mathrm{BpscQ}, \mathrm{BpscR}$, BpscS, Orf1 and Orf2 have been discussed previously [14]. $\mathrm{BpscN}$ had one of the highest sequence identities with its $R$. solanacearum homologue $(\mathrm{HrcN})$ and contains an ATP-binding 'A' consensus sequence ( $\mathrm{P}$ loop; 182APAGVGKS) and an ATP synthase $\alpha \beta$ signature (362PAIDVLGSLS) typical of TTS-associated ATPases. BpscJ has a prokaryotic membrane lipoprotein lipid attachment site (23-VLALSVLLAGC) similar to those found in the equivalent $R$. solanacearum ( $\mathrm{HrcJ})$ and Yersinia (YscJ) proteins. As was the case with their $R$. solanacearum homologues [20], hydropathy analysis predicted that Orf7 and BpscL were highly hydrophilic.

Analysis with the programme MOTIFS indicated that the predicted protein BpscC contains a protein $\mathrm{D}$ signature common to type II and type III secretion outer-membrane secretin proteins (536-GQSLLIAGY STDKRANGVAGVPWLSKIPLLGALF). BpscC also contained a putative signature characteristic of bacterial regulatory proteins of the LysR family. Proteins of the LysR family possess a potential 'helix-turn-helix' DNA-binding motif in their N-terminal section. The amino acid sequence pattern used to detect these proteins is a consensus sequence derived from the complete helix-turn-helix motif and the next 10 residues. In $\mathrm{BpscC}$, the consensus sequence was not detected in the N-terminal region and therefore is probably not significant.

It has been predicted that the N-terminal half of secretins faces the periplasm and this region is not well conserved [25]. The $\mathrm{C}$-terminal region ( $\beta$ domain) is more conserved and is predicted to contain several transmembrane $\beta$-strands whose likely location is the outer membrane. BpscC aligns strongly with homologues in the conserved $\mathrm{C}$-terminal region and contains the consensus motif (V,I)PXL(S,G)XIPXXGXLF common to proteins of this family [26]. BpscV aligns particularly strongly with the N-terminal region of $R$. solanacearum $\mathrm{HrcV}$. In $\mathrm{HrcV}$ and homologues this region of the protein is predicted to be hydrophobic, with membrane-associated helices [27]. The less conserved C-terminal region is hydrophilic. BpscT aligns with the $R$. solanacearum secreton inner-membrane protein HrcT. Proteins of this family are highly hydrophobic, containing a number of membraneassociated helices.
Downstream of $b p s c C$ lies a region containing a 264-bp imperfect triple direct repeat sequence that marks the boundary between the gene cluster with homology to $R$. solanacearum and Xanthomonas spp. and several putative ORFs (Table 4) likely to include genes encoding secreted proteins if the B. pseudomallei TTS gene cluster continues its resemblance to that of the plant pathogens. It is possible to identify an ORF within this repeated region. If genuine, this ORF, transcribed in the same direction as bpscC and orf9, would encode a glycine-rich 461 residue peptide with predicted mol. wt and pI values of $45764 \mathrm{Da}$ and 4.16, respectively, and no significant homology to known proteins. As the repeated sequences are in-frame with each other, any such peptide would contain regions of repeated amino acid sequences.

Orf9 is predicted to be a largely hydrophilic protein with a C-terminal hydrophobic $\alpha$-helical domain. BLAST searches identified homology with $P$. syringae HrpK (32\% identity $/ 46 \%$ positives between residues over 338 amino acid residues (94-418); accession no. AF232004), a hydrophilic protein for which a function has yet to be ascribed. However, P. syringae HrpK does not contain a C-terminal hydrophobic domain. BLAST searches indicated that Orf10 shared best homology with acetyltransferases. The highest match was 35\% identity and $51 \%$ positives over 65 amino acid residues (Orf10 73-132) against a ribosomal-protein-alanine Nacetyltransferase of Bacillus halodurans (accession no. AP001509). BLAST searches failed to identify any significant homology between Orf11 and known proteins. MOTIFS indicated that Orf11 contains a putative actinin-type actin-binding domain signature $((\mathrm{E}, \mathrm{Q}) \times 2(\mathrm{~A}, \mathrm{~T}, \mathrm{~V})(\mathrm{F}, \mathrm{Y}) \times 2 \mathrm{WxN})$. In most proteins identified as possessing this motif, including $\alpha$-actinin, the signature resides within the $\mathrm{N}$-terminal region. An exception to this rule is fimbrin, where the signature is located in duplicate in the C-terminal region. In Orf11, the signature is located between residues 551 and 560 . Orf11 also contains a putative ATP/GTP-binding site P-loop; $(\mathrm{A}, \mathrm{G}) \times 4 \mathrm{GK}(\mathrm{S}, \mathrm{T})$ - typical of kinases, ADPribosylating factors and a number of proteins involved in secretion.

Downstream of Orf3 lie other potential genes. The predicted protein of Orf12 shows strong homology to $\mathrm{HpaB}$ (accession no. BAB07868), a protein of the

Table 4. Predicted properties of putative proteins encoded by ORFs downstream of bpscC and orf 3

\begin{tabular}{ccccl}
\hline ORF & Length & Mol. wt (Da) & pI & Properties/BLASTP matches \\
\hline 9 & $602(641)$ & $61412(67678)$ & $4.33(4.98)$ & $\begin{array}{l}\text { Homology with Pseudomonas syringae HrpK (properties indicated in } \\
\text { parentheses) }\left(338 \text { residues; } E=1 \times \mathrm{e}^{-29}\right)\end{array}$ \\
10 & 161 & 17852 & 7.10 & $\begin{array}{l}\text { Some homology with acetyltransferases } \\
\text { Actinin-type actin-binding domain signature; glycine rich; ATP/GTP-binding } \\
\text { site motif A (P-loop) }\end{array}$ \\
11 & 1287 & 9.34 & 4.18 & $\begin{array}{l}\text { Homology with Xanthomonas oryzae pv. oryzae HpaB }(151 \text { residues; } \\
\left.E=1 \times \mathrm{e}^{-36}\right) \\
\text { Homology with Serratia entomophila SepC }\left(327 \text { residues; } E=2 \times \mathrm{e}^{-27}\right)\end{array}$ \\
13 & 151 & 16847 & 10.40 &
\end{tabular}


Xanthomonas spp. TTS system. These proteins are small and acidic, properties suggesting that they may function as chaperones [12]. Further downstream lies Orf13, which has homology to SepC, a potential insect toxin of Serratia entomophila [28].

\section{Dot-blot and PCR assays}

Table 1 summarises the data obtained by dot-blot and PCR assays to determine the presence of different TTS genes in various strains of $B$. pseudomallei and $B$. thailandensis. Five TTS1 loci were targeted by dot-blot and PCR assays. Only dot-blot data are presented for two of the loci because PCR amplification with the primers PM84/PM95 (for orf4/orf5) and PMXBA3/ PMBAM (for the gap between orfl and $b p s c Q$ ) with the various genomic DNAs proved to be difficult. However, in both cases it was possible to produce amplicons for DIG-labelling to make probes for dot-blot analysis. Fig. 2 shows examples of PCR assay results. In all cases the $B$. pseudomallei strains tested positive for the presence of TTS-related DNA whereas B. thailandensis strains were negative. Strain E27, which had previously proved positive for orf2/bpscD by both PCR and dot-blot assays [15], was negative for the loci tested in this study. Occasionally, a faint amplicon of the correct size was visible when primers PM98/PM99 (for bpscJ) were used to amplify strain E27 genomic DNA. However, dot-blot analysis suggested that this gene is not present in strain E27, or is significantly different from the bpsc genes present in B. pseudomallei strains.

\section{RT-PCR}

The conditions required for expression of TTS1 genes in B. pseudomallei were not known. In Pseudomonas aeruginosa, the addition of NTA to growth media induces TTS genes [29]. The initial approach in the present study involved culturing the bacteria at $37^{\circ} \mathrm{C}$ in the presence or absence of NTA. Two primer combinations were used to screen for gene expression from putative genes either side of the repeat sequence region of the gene cluster. The three genes targeted were bpscJ, orfl0 and orf11. The $f l i C$ gene was targeted as a positive control. In both strains E503 and E27, grown with and without NTA, the $f i C$ gene could be amplified from cDNA. All of the controls where reversetranscriptase was not used were negative, indicating that there was no residual genomic DNA in the cDNA samples (Fig. 3). RT-PCR amplicons for bpscJ, orf10 and $\operatorname{orf11}$ were obtained with strain E503 samples only. This occurred with and without NTA, indicating that NTA is not required for the expression of these genes in B. pseudomallei.

\section{TTS2 and comparisons with B. mallei}

Comparisons of B. pseudomallei E503 TTS1 sequences with those available through the $B$. pseudomallei genome sequence project indicated that there are sequence variations (typically $1-2 \%$ ). Such comparisons also suggested that there was a second TTS gene cluster in this bacterium. A cluster of genes homologous to those encoding BpscU, BpscV, Orf1, BpscQ, BpscR, BpscS, Orf2, BpscD, Orf3 and Orf4, could be identified on the same 9315-bp sequence contig. A comparison of this cluster (TTS2) with B. mallei genome sequence project data indicated the presence of an equivalent gene cluster ( $99 \%$ sequence identity), and further suggested that genes encoding Orf5, BpscJ, Orf6, BpscL and $\mathrm{BpscN}$ were also present in the cluster. Further analysis of another B. pseudomallei sequence
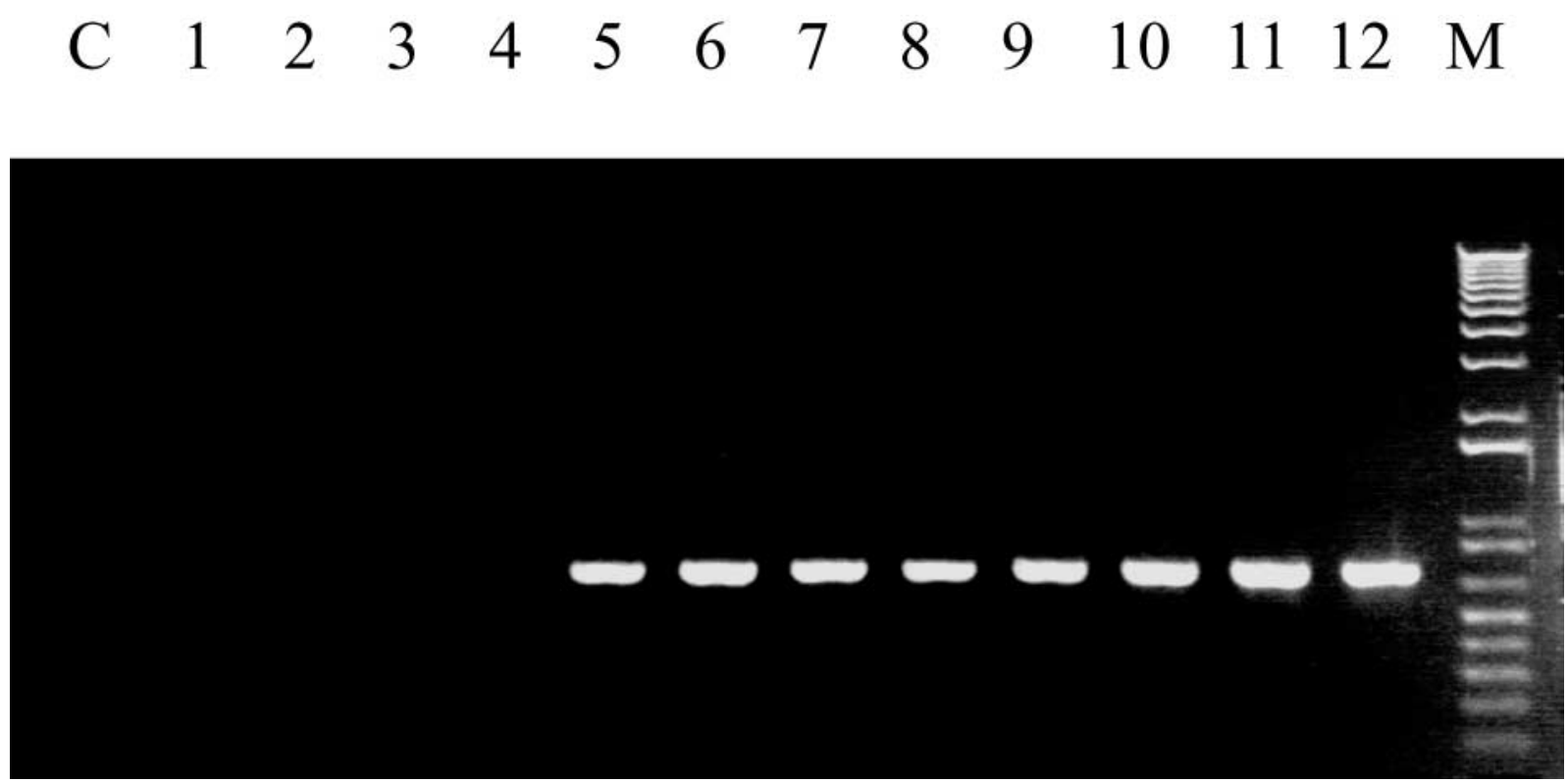

Fig. 2. PCR assays for the presence of TTS1 genes. Examples of results of PCR assays for the presence of orf11 are shown. The strains tested were: 1, E27; 2, E125; 3, E111; 4, E32; 5, E506; 6, E505; 7, E504; 8, E503; 9, E8; 10, E25; 11, 576; 12, 204. Lanes 1-4, results for $B$. thailandensis strains; 5-12, results for $B$. pseudomallei strains. C, reagent control; M, 1-kb Plus DNA ladder size marker (Life Technologies, Renfrewshire). 
a
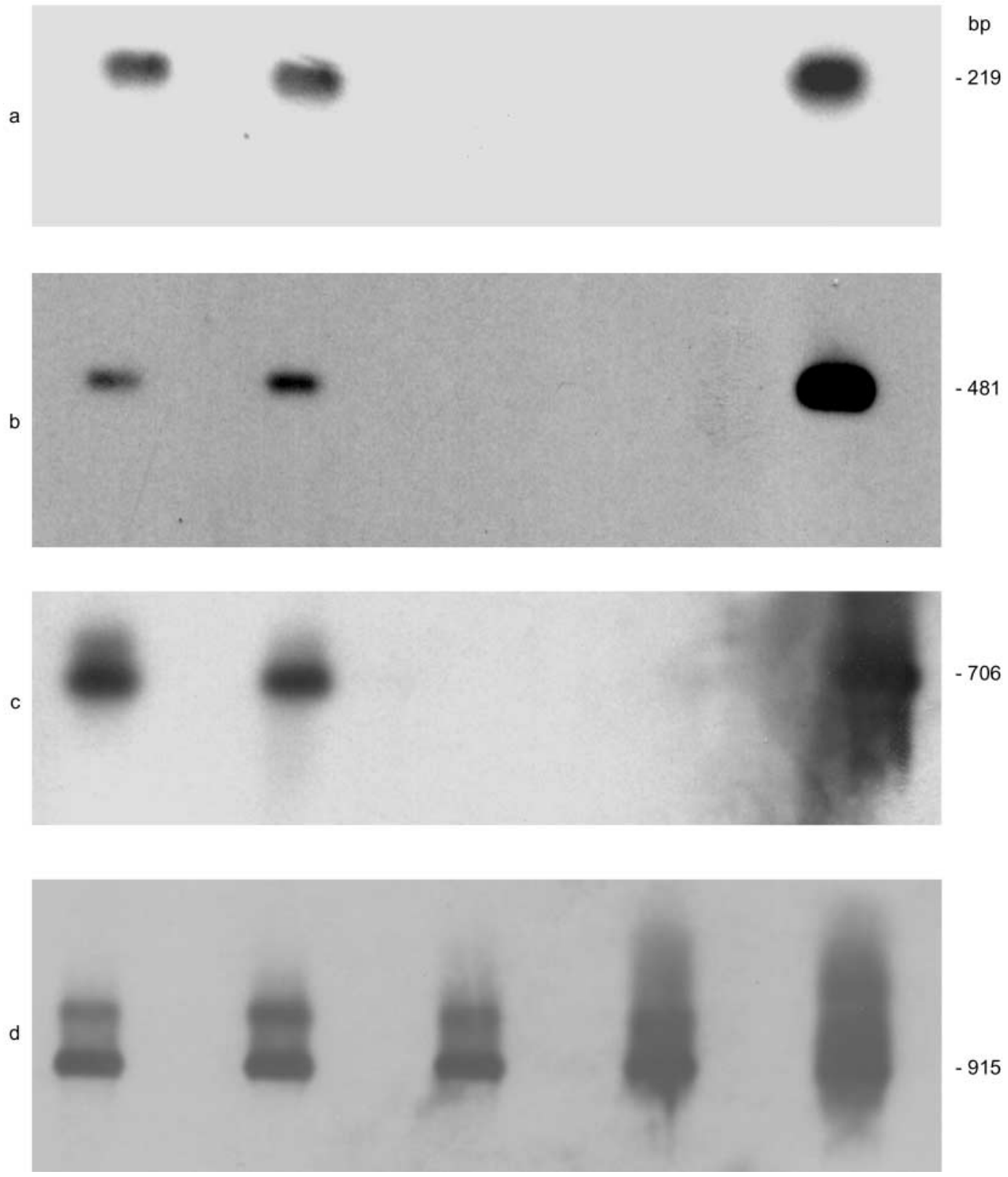

Fig. 3. RT-PCR results for detection of expression of $\operatorname{orfl0}(\mathbf{a}), \operatorname{bpscJ}(\mathbf{b}), \operatorname{orfl1}(\mathbf{c})$ and $f i C$ (d) are shown. The figure comprises Southern blot hybridisation data from four gels. In each case the lanes refer to the following: 1, strain E503 grown at $37^{\circ} \mathrm{C}$ in the absence of NTA; 3, strain E503 grown at $37^{\circ} \mathrm{C}$ in the presence of NTA; 5, strain E27 grown at $37^{\circ} \mathrm{C}$ in the absence of NTA; 7 , strain E27 grown at $37^{\circ} \mathrm{C}$ in the presence of NTA; 2, 4, 6, 8, controls lacking reverse-transcriptase for equivalent reactions to preceding lanes; 9, genomic DNA controls.

contig, overlapping with the $B$. mallei sequence, suggests that the TTS2 gene cluster resembles TTS1 in gene organisation from bpscT-orf12, but that an equivalent gene to orf8 lies downstream of the TTS2 orf 12 and that the equivalent gene to $b p s c C$ is several kilobases further downstream. Indeed, there are two possible bpscC equivalents ( $b p s c C 2 a$ and $b p s c C 2 b$ ) separated by a cluster of genes with homology to genes involved in type IV pilus production (Fig. 1). PCR assays were used to screen three B. pseudomallei and three $B$. thailandensis strains, and indicated that the TTS2 gene bpscU2 is present in both B. pseudomallei and B. thailandensis (data not shown). There was no evidence for the presence of the TTS1 gene cluster in $B$. mallei from orf11 to orf12 (at the time the search was conducted the data were estimated to represent at least three-fold coverage of the genome). However, a BLASTX search with Orf13 indicated its presence in B. mallei (99\% sequence identity). Further sequence analysis confirmed that orf13 and DNA directly downstream are present in B. mallei but upstream of orf13 the sequence similarity ends (between orf12 and orf13). A PCR assay for $\operatorname{orfl} 13$ was conducted on a selection of $B$. pseudomallei and $B$. thailandensis strains. The results indicate that unlike B. mallei and B. pseudomallei, $B$. thailandensis strains lack orf13 (data not shown). 


\section{Discussion}

The TTS1 sequenced region of the B. pseudomallei E503 genome reported in this paper contains numerous putative genes with homology to TTS system genes reported previously and is highly likely to encode such a system. The overall $\mathrm{G}+\mathrm{C}$ content $(63 \mathrm{~mol} \%)$ is similar to the value reported previously for the region spanning orf1-orf2 [14] and is lower than the value estimated for this organism by the B. pseudomallei sequencing group (65 mol\%; ftp://ftp.sanger.ac.uk/pub/ pathogens/bps). However, the difference in $\mathrm{G}+\mathrm{C}$ content is marginal compared with some reported for TTS PIs in other bacteria [12]. Although the genes encoding TTS systems are generally clustered together, the order of genes varies between bacteria. Whereas proteins involved in the formation of the secretion structure, or secreton, tend to be conserved throughout TTS systems, other proteins are apparent only in related groups of bacteria, or are unique to a particular TTS system. Often genes are conserved in relative location between bacteria whilst exhibiting low sequence homologies [12,13]. The organisation of secreton genes in the B. pseudomallei TTS1 cluster strongly resembles that seen in the plant pathogens $R$. solanacearum and Xanthomonas spp. The only significant difference stems from the fact that in Xanthomonas spp., $h r p X v$, the equivalent of the genes encoding the putative transcriptional regulators $\mathrm{HrpB}$ (in $R$. solanacearum) and Orf8 (B. pseudomallei), is not located between the genes encoding BpscC and BpscT or homologues, but lies in an unlinked position [30].

The regions downstream of $b p s c C$ and its equivalents differ significantly between $B$. pseudomallei TTS1, $R$. solanacearum and Xanthomonas spp. In $R$. solanacearum and Xanthomonas spp., this domain marks the boundary between genes encoding proteins with largely structural roles in the formation of a secreton and those that encode the secreted products. Therefore, it would seem reasonable to postulate that ORFs in B. pseudomallei lying in an equivalent location may encode potential type III-secreted proteins or accessory proteins associated with secretion. In B. pseudomallei, the boundary is marked by the presence of a repeated sequence, which may be indicative of a recombination event that has linked the structural genes of the TTS system with genes encoding effector or other proteins specific to $B$. pseudomallei.

PCR and dot-blot assays in this study confirmed the association between TTS genes and an $\mathrm{Ara}^{-}$phenotype reported previously [15]. The earlier observations that one B. thailandensis strain (E27) was carrying some TTS1 system-related DNA were not further substantiated. The results obtained previously for the orf $2 /$ bpscD region, and the reproducible presence of a faint band following PCR amplification with the PM98/ PM99 primer set (for bpscJ) cannot simply be explained by the presence of TTS2 in B. thailandensis because none of the primers match the TTS2 sequence sufficiently well. There may be DNA in $B$. thailandensis strain E27 that is related to B. pseudomallei TTS1 DNA. If this is the case, the equivalent genes in strain E27 are sufficiently different from those in B. pseudomallei to ensure that detection is not possible by dotblot hybridisation in most cases. The alternative explanation is that strain E27 contains DNA equivalent to some but not all the B. pseudomallei TTS1 gene cluster. The RT-PCR assays used in this study did not lead to the detection of any expression of TTS1 genes in E27. The problems associated with two of the PCR assays in this study may well be related to minor sequence variations between strains, causing primer mismatching.

In many cases, although TTS is triggered in situ by cell contact, growth conditions can induce expression of TTS genes. Secretion of ExoS and other proteins by $P$. aeruginosa can be induced artificially by growth in the presence of the chelator NTA [29]. The present observations indicate that expression of the B. pseudomallei TTS system is detectable during growth in Luria broth at $37^{\circ} \mathrm{C}$. The addition of NTA to growth media was not required for TTS gene expression. The data presented here do not include quantification of gene expression, and there may be growth conditions that lead to higher levels of expression. However, it is clear that there was some expression of TTS genes in nutrient-rich media at $37^{\circ} \mathrm{C}$. There are several examples of artificial induction of TTS, including the TTS systems of Yersinia spp. (induced by growth in media containing low levels of calcium ions [31]), Shigella spp. (in which chemical compounds, including Congo red, Evans blue and direct orange, induce secretion of Ipa proteins [32]) and Salmonella enterica serovar Typhimurium (in which SPI2 genes can be induced by $\mathrm{Mg}^{2+}$ deprivation and phosphate starvation [33]). However, temperature induction of TTS without the requirement for additional media supplements is not unprecedented. In Bordetella bronchiseptica, TTS is under the control of the BvgAS regulon, and can be induced in response to growth at $37^{\circ} \mathrm{C}\left(\mathrm{Bvg}^{+}\right)$. In contrast, growth at or below $26^{\circ} \mathrm{C}$, or in the presence of high concentrations of nicotinic acid or $\mathrm{MgSO}_{4}$ $\left(\mathrm{Bvg}^{-}\right)$, excludes the production of almost all of the known virulence factors in Bordetella spp., including TTS [34].

There is considerable homology between the predicted Orf8 protein and $R$. solanacearum $\mathrm{HrpB}$, the positive regulator responsible for regulating expression of TTS operons [20,21], and a member of the AraC family. Putative binding sites for such regulatory proteins are difficult to identify from sequence data. A consensus sequence (TTTTaGYcTtTat, in which nucleotides conserved in $\geqslant 60 \%$ of sequences are shown in capital letters) has been reported for VirF binding sites in Yersinia spp. [35]. The present study found a sequence conforming to this consensus upstream of the orf8 
gene. The presence of a putative VirF-like binding site suggests that, as in $R$. solanacearum, the B. pseudomallei TTS gene cluster may encode a transcriptional activator that is autoregulated. In Xanthomonas spp. $\mathrm{HrpXv}$, the equivalent AraC-like positive regulatory protein, is not autoregulated [30].

In $R$. solanacearum the hrp genes are expressed in minimal media but repressed in nutrient-rich media [36], which suggests that the regulatory system differs considerably from that of $B$. pseudomallei TTS1. The fact that the latter system is induced in nutrient-rich media at $37^{\circ} \mathrm{C}$ may be indicative of a system designed for activity in mammals rather than in plants. A number of candidate ORFs for secreted proteins have been identified by relative location compared to the TTS PIs of $R$. solanacearum and Xanthomonas spp. RT-PCR indicated that orf10 and orf11 are expressed, suggesting that this region does contain functional, expressed genes. There are putative ribosome-binding sites (AGGA) upstream of orf9 and orf10. The end of orf9 is separated from the beginning of orf10 by only $24 \mathrm{bp}$. Therefore, it seems likely that orf9, orf10 and orfl1 form an operon. Although there is a potential ORF within the repeat region, and there are potential ribosome-binding sequences upstream of this ORF (5'GGAGGCGGAA); without evidence to the contrary it seems unlikely that this does encode a functional protein. Any such protein would contain the same sequence repeated imperfectly three times with short intervening gaps.

Only one of the predicted protein sequences derived from these ORFs was significantly homologous to proteins identified in other TTS systems. Orf9 had homology to the HrpK protein of $P$. syringae, a protein of unknown function. This homology did not include the C-terminal region, which suggests that there may be functional differences between these proteins. Although Orf10 shared some homology with a number of bacterial acetyltransferases, $E$ values were much higher than the level generally considered as indicating significance $\left(E<1 \times \mathrm{e}^{-5}\right)$. Orf11 is a large protein, which shares no significant homology to any proteins in the database, but contains a number of potential motifs. There is evidence that B. pseudomallei is a facultative intracellular pathogen, capable of multiplying within various cell types [37]. It has been demonstrated that, after internalisation, the organism can escape from membrane-bound phagosomes into the cytoplasm [37-39]. More recently it has been shown that $B$. pseudomallei can induce cell fusion leading to the formation of multinucleated giant cells and actinassociated membrane protrusion in phagocytic and nonphagocytic cell lines, potentially enabling the pathogen to spread between cells [40]. It is tempting to speculate that type III-secreted proteins may be implicated in such interactions with actin. However, neither ActA, SipA nor SipC contain the actinin-type actin-binding domain signature sought by the programme MOTIFS, and the significance of the signature identified in Orf11 is difficult to assess without evidence of activity. A number of protein families have the ATP/GTP-binding site motif identified in Orf11. Amongst the most interesting of these are members of the ADP-ribosylation factors family and kinases.

Downstream from orf11, following a gap of $310 \mathrm{bp}$, there is DNA which when translated in the opposite orientation to orfl1 gives a partial predicted protein sequence with strong homology $(50 \%$ identity $/ 65 \%$ similarity over 251 amino acids; BLASTP $E$ value of $3 \times \mathrm{e}^{-58}$ ) to a transposase of $R$. metallidurans (AF236817). Residual transposon or IS sequences are a feature of PIs [12] and the presence of such a sequence strongly suggests that the TTS-associated gene cluster ends at this point, and that a putative transposase may have played a role in the acquisition of this gene cluster by B. pseudomallei E503. Interestingly, the high levels of sequence identity between strain E503 TTS1 and the equivalent region of the strain chosen for genome sequencing, K96243, ends between orf11 and the putative transposase gene. Indeed, BLAST searches failed to identify the strain E503 transposase gene in K96243 or B. mallei, although there are sequences with homology to other transposases downstream of orf11 in K96243 (transposase of $T n 1721 / \operatorname{Tn} 501, E=3 \times \mathrm{e}^{-22}$, over 117 amino acids). However, the K96243 sequence contains a stop codon within the matching region, suggesting either that the genome data is erroneous or that a mutation has occurred that would render the gene inactive. This observation does suggest that there may be DNA sequence differences between strains at the edge of the TTS1 gene cluster, and that there are variations in transposon or IS sequences between strains of $B$. pseudomallei.

With TTS1 predicted proteins as probes in silico, a second TTS gene cluster (TTS2) was identified in both $B$. pseudomallei and B. mallei. Analysis indicates that $B$. pseudomallei and $B$. mallei both carry TTS2 genes whereas only B. pseudomallei carries the TTS1 gene cluster. In addition, a PCR assay for detection of bpscU2 indicated that at least part of TTS2 is present in B. thailandensis. Recently, it has become apparent that there is a third TTS system gene cluster (tentatively named TTS3). TTS3 includes genes with homology to some of the Salmonella Inv/Spa secreted virulence factors [41] and is present in B. pseudomallei and $B$. mallei. A PCR assay to screen for the putative gene encoding a protein with homology to Salmonella SopE showed evidence to suggest that TTS3 DNA is also present in B. thailandensis (data not shown). Thus, only the TTS1 system defines a major difference between $B$. pseudomallei and both B. thailandensis and $B$. mallei. One boundary marking the variation between B. mallei and B. pseudomallei lies between orf 12 and orf13 in TTS1. At this stage, it is not clear whether orf 13 should be considered part of the TTS1 gene cluster. Orf13 is homologous to the C-terminal region 
of the putative insect toxin SepC. Hurst et al. [28] demonstrated homology between SepC and other insect toxins. However, those regions common to insect toxins were not the same as the region of best homology with Orf13. Whether Orf13 is involved in TTS or not, it may be a gene of interest because of its presence in $B$. mallei and B. pseudomallei but absence from $B$. thailandensis. The TTS2 genes are organised in a similar fashion to the TTS1, $R$. solanacearum and Xanthomonas spp. TTS gene clusters suggesting common ancestry for all these systems. Although the $B$. pseudomallei TTS1 and TTS2 genes are most similar to each other, there has been considerable divergence suggesting that the two TTS systems may have different functions. Although the actual role of TTS during infection with $B$. pseudomallei has yet to be resolved, the likelihood is that, as in all other cases, TTS makes an important contribution to the pathogenicity of this bacterium.

This work was funded by the award of a grant to C.W. and C.A.H. from the Wellcome Trust (reference 58935). Whole genome sequencing of $B$. mallei and $B$. pseudomallei was accomplished with support from the National Institute of Health, USA and Beowulf Genomics, respectively.

\section{References}

1. Dance DA. Melioidosis: the tip of the iceberg? Clin Microbiol Rev 1991; 4: 52-60.

2. Leelarasamee A, Bovornkitti S. Melioidosis: review and update. Rev Infect Dis 1989; 11: 413-425.

3. Chaowagul W, Suputtamongkol Y, Dance DAB, Rajchanuvong A, Pattara-arechachai J, White NJ. Relapse in melioidosis: incidence and risk factors. $J$ Infect Dis 1993; 168: 1181-1185.

4. Currie BJ, Fisher DA, Anstey NM, Jacups SP. Melioidosis: acute and chronic disease, relapse and re-activation. Trans $R$ Soc Trop Med Hyg 2000; 94: 301-304.

5. Brett PJ, DeShazer D, Woods DE. Burkholderia thailandensis sp. nov., a Burkholderia pseudomallei-like species. Int J Syst Bacteriol 1998; 48: 317-320.

6. Smith MD, Angus BJ, Wuthiekanun V, White NJ. Arabinose assimilation defines a nonvirulent biotype of Burkholderia pseudomallei. Infect Immun 1997; 65: 4319-4321.

7. Winstanley C, Hales BA, Corkill JE, Gallagher MJ, Hart CA. Flagellin gene variation between clinical and environmental isolates of Burkholderia pseudomallei contrasts with the invariance among clinical isolates. J Med Microbiol 1998; 47: $689-694$

8. Wajanarogana S, Sonthayanon P, Wuthiekanun V, Panyim S, Simpson AJH, Tungpradabkul S. Stable marker on flagellin gene sequences related to arabinose non-assimilating pathogenic Burkholderia pseudomallei. Microbiol Immunol 1999; 43: 995-1001.

9. Dharakul T, Tassaneetrithep B, Trakulsomboon S, Songsivilai S. Phylogenetic analysis of $\mathrm{Ara}^{+}$and $\mathrm{Ara}^{-}$Burkholderia pseudomallei isolates and development of a multiplex PCR procedure for rapid discrimination between the two biotypes. J Clin Microbiol 1999; 37: 1906-1912.

10. Chaiyaroj SC, Kotrnon K, Koonpaew S, Anantagool N, White NJ, Sirisinha S. Differences in genomic macrorestriction patterns of arabinose-positive (Burkholderia thailandensis) and arabinose-negative Burkholderia pseudomallei. Microbiol Immunol 1999; 43: 625-630.

11. Reckseidler SL, Deshazer D, Sokol PA, Woods DE. Detection of bacterial virulence genes by subtractive hybridization: identification of capsular polysaccharide of Burkholderia pseudomallei as a major virulence determinant. Infect Immun 2001; 69: 34-44

12. Hueck CJ. Type III protein secretion systems in bacterial pathogens of animals and plants. Microbiol Mol Biol Rev 1998;
62: $379-433$.

13. Winstanley C, Hart CA. Type III secretion systems and pathogenicity islands. J Med Microbiol 2001; 50: 116-126.

14. Winstanley C, Hales BA, Hart CA. Evidence for the presence in Burkholderia pseudomallei of a type III secretion systemassociated gene cluster. J Med Microbiol 1999; 48: 649-656.

15. Winstanley C, Hart CA. Presence of type III secretion genes in Burkholderia pseudomallei correlates with $\mathrm{Ara}^{-}$phenotypes. $J$ Clin Microbiol 2000; 38: 883-885.

16. Pearson WR, Lipman DJ. Improved tools for biological sequence comparison. Proc Natl Acad Sci USA 1988; 85: 2444-2448.

17. Kyte J, Doolittle RF. A simple method for displaying the hydropathic character of a protein. J Mol Biol 1982; 157: 105-132.

18. Eisenberg D, Weiss RM, Terwilliger TC. The hydrophobic moment detects periodicity in protein hydrophobicity. Proc Natl Acad Sci USA 1984; 81: 140-144.

19. Altschul SF, Madden TL, Schäffer AA et al. Gapped BLAST and PSI-BLAST: a new generation of protein database search programs. Nucleic Acids Res 1997; 25: 3389-3402.

20. Van Gijsegem F, Gough C, Zischek C et al. The hrp gene locus of Pseudomonas solanacearum, which controls the production of a type III secretion system, encodes eight proteins related to components of the bacterial flagellar biogenesis complex. Mol Microbiol 1995; 15: 1095-1114.

21. Guéneron $\mathrm{M}$, Timmers ACJ, Boucher C, Arlat M. Two novel proteins, PopB, which has functional nuclear localization signals, and PopC, which has a large leucine-rich repeat domain, are secreted through the Hrp-secretion apparatus of Ralstonia solanacearum. Mol Microbiol 2000; 36: 261-277.

22. Fenselau S, Bonas U. Sequence and expression analysis of the hrpB pathogenicity operon of Xanthomonas campestris $p v$. vesicatoria which encodes eight proteins with similarity to components of the Hrp, Ysc, Spa, and Fli secretion systems. Mol Plant Microbe Interact 1995; 8: 845-854.

23. Huguet E, Hahn K, Wengelnik K, Bonas U. hpaA mutants of Xanthomonas campestris $p v$. vesicatoria are affected in pathogenicity but retain the ability to induce host-specific hypersensitive reaction. Mol Microbiol 1998; 29: 1379-1390.

24. Zhu W, Magbanua MM, White FF. Identification of two novel hrp-associated genes in the hrp gene cluster of Xanthomonas oryzae pv. oryzae. J Bacteriol 2000; 182: 1844-1853.

25. Guilvout I, Hardie KR, Sauvonnet N, Pugsley AP. Genetic dissection of the outer membrane secretin PulD: are there distinct domains for multimerization and secretion specificity? $J$ Bacteriol 1999; 181: 7212-7220.

26. Genin S, Boucher CA. A superfamily of proteins involved in different secretion pathways in gram-negative bacteria: modular structure and specificity of the N-terminal domain. Mol Gen Genet 1994; 243: 112-118.

27. Gough CL, Genin S, Lopes V, Boucher CA. Homology between the HrpO protein of Pseudomonas solanacearum and bacterial proteins implicated in a signal peptide-independent secretion mechanism. Mol Gen Genet 1993; 239: 378392.

28. Hurst MRH, Glare TR, Jackson TA, Ronson CW. Plasmidlocated pathogenicity determinants of Serratia entomophila, the causal agent of amber disease of grass grub, show similarity to the insecticidal toxins of Photorhabdus luminescens. $J$ Bacteriol 2000; 182: 5127-5138.

29. Yahr TL, Mende-Mueller LM, Friese MB, Frank DW Identification of type III secreted products of the Pseudomonas aeruginosa exoenzyme S regulon. J Bacteriol 1997; 179: $7165-7168$.

30. Wengelnik K, Bonas U. HrpXv, an AraC-type regulator, activates expression of five of the six loci in the hrp cluster of Xanthomonas campestris pv. vesicatoria. J Bacteriol 1996 178: $3462-3469$.

31. Cornelis GR, Boland A, Boyd AP et al. The virulence plasmid of Yersinia, an antihost genome. Microbiol Mol Biol Rev 1998; 62: $1315-1352$.

32. Bahrani FK, Sansonetti PJ, Parsot C. Secretion of Ipa proteins by Shigella flexneri: inducer molecules and kinetics of activation. Infect Immun 1997; 65: 4005-4010.

33. Deiwick J, Nikolaus T, Erdogan S, Hensel M. Environmental regulation of Salmonella pathogenicity island 2 gene expression. Mol Microbiol 1999; 31: 1759-1773.

34. Yuk MH, Harvill ET, Miller JF. The BvgAS virulence control 
system regulates type III secretion in Bordetella bronchiseptica. Mol Microbiol 1998; 28: 945-959.

35. Wattiau P, Cornelis GR. Identification of DNA sequences recognized by VirF, the transcriptional activator of the Yersinia yop regulon. J Bacteriol 1994; 176: 3878-3884.

36. Arlat M, Gough CL, Zischek C, Barberis PA, Trigalet A, Boucher CA. Transcriptional organization and expression of the large hrp gene cluster of Pseudomonas solanacearum. Mol Plant Microbe Interact 1992; 5: 187-193.

37. Jones AL, Beveridge TJ, Woods DE. Intracellular survival of Burkholderia pseudomallei. Infect Immun 1996; 64: 782-790.

38. Pruksachartvuthi S, Aswapokee N, Thankerngpol K. Survival of Pseudomonas pseudomallei in human phagocytes. J Med
Microbiol 1990; 31: 109-114.

39. Harley VS, Dance DAB, Drasar BS, Tovey G. Effects of Burkholderia pseudomallei and other Burkholderia species on eukaryotic cells in tissue culture. Microbios 1998; 96: 71-93.

40. Kespichayawattana W, Rattanachetkul S, Wanun T, Utaisincharoen P, Sirisinha S. Burkholderia pseudomallei induces cell fusion and actin-associated membrane protrusion: a possible mechanism for cell-to-cell spreading. Infect Immun 2000; 68: 5377-5384.

41. Attree O, Attree I. A second type III secretion system in Burkholderia pseudomallei: who is the real culprit? Microbiol 2001; 147: 3197-3199. 